\title{
Characterization of electronic properties of natural type IIb diamonds
}

\author{
V.I. Zubkov $^{1 \mathrm{a})}$, A.V. Solomnikova ${ }^{1}$, J.E. Post ${ }^{2}$, E. Gaillou ${ }^{2,3}$, and J.E. Butler ${ }^{1,2}$ \\ ${ }^{1}$ St. Petersburg State Electrotechnical University (LETI), Professor Popov Street 5, 197376 St. Peters- \\ burg, Russia \\ ${ }^{2}$ National Museum of Natural History (NMNH), P.O. Box 37012 Smithsonian Inst., Washington D.C., \\ 20013-7012 \\ ${ }^{3}$ MINES ParisTech, PSL Research University, Musée de Minéralogie, 60 boulevard Saint-Michel, \\ 75006 Paris, France
}

\begin{abstract}
Precision admittance spectroscopy measurements were carried out over wide temperature and frequency ranges for a set of natural single crystal type IIb diamond samples. Peaks of conductance spectra vs. temperature and frequency were used to compute the Arrhenius plots, and activation energies were derived from these plots. The capacitance-voltage profiling was used to estimate the majority charge carrier concentration and its distribution into depth of the samples. Apparent activation energies between 315 and $325 \mathrm{meV}$ and the capture cross section of about $10^{-13} \mathrm{~cm}^{2}$ were found for samples with uncompensated boron concentrations in the range of 1 to $5 \times 10^{16} \mathrm{~cm}^{-3}(0.06-0.3 \mathrm{ppm})$. The obtained boron concentrations are in good coincidence with FTIR results for the samples. Also, a reason for the difference between the observed admittance activation energy and the previously reported ionization energy for the acceptor boron in diamond $(0.37 \mathrm{eV})$ is proposed.
\end{abstract}

\section{Graphical abstract}

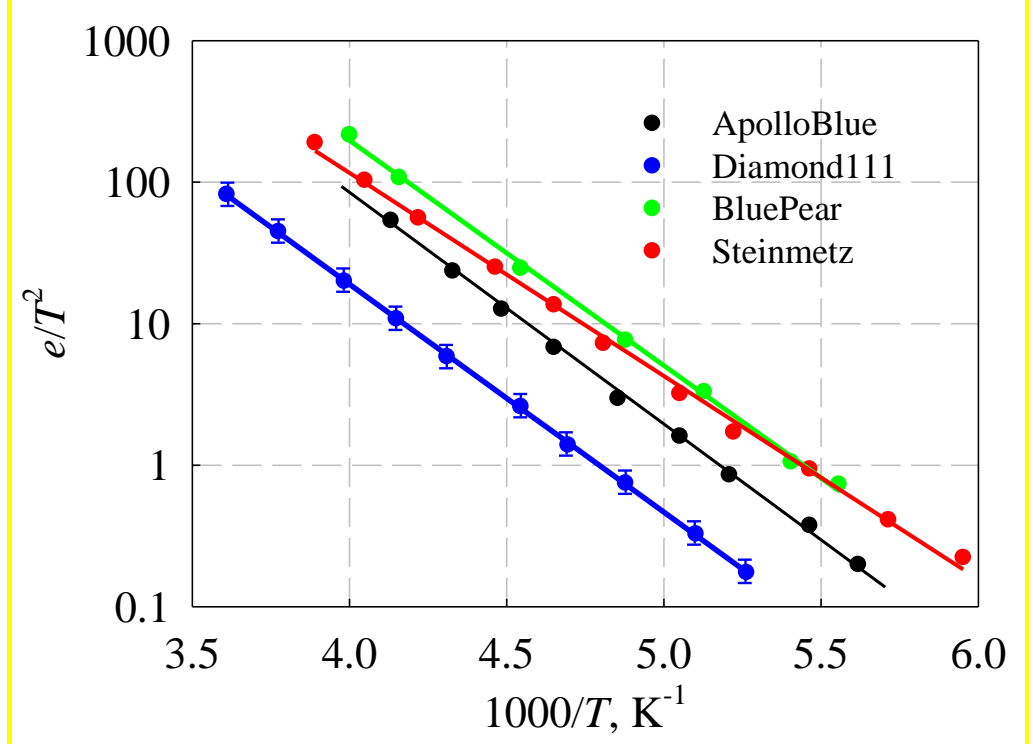

âVZubkovspb@mail.ru 


\section{Key words}

Natural diamond, boron, admittance spectroscopy, charge carrier concentration, activation energy.

\section{Introduction}

Natural (mined) diamonds may contain several impurity elements in the crystal lattice which can affect their optical, electrical and other physical properties.[1] Nitrogen is the most common impurity found and can occur as a substitution atom in the lattice, or as more complicated clusters of nitrogen atoms and lattice vacancies. As a result, natural diamonds are generally classified as Type I, if they contain sufficient nitrogen as detected by infrared absorption spectroscopy (IR), and Type II, if nitrogen related defects are undetectable by IR. Type II diamonds, with no IR detectable nitrogen, generally make up less than $2 \%$ of all mined diamonds. Other impurity atoms found in natural diamonds are hydrogen, boron, and silicon. More complicated mineral inclusions are also found in natural diamonds and are important indicators of the diamond genesis environment. The type II class of diamonds is further divided into type IIa, where neither nitrogen nor boron is detectable by IR, and type IIb, where only boron related defects are detectable by IR.[1]

Amongst the rarest and most valuable of natural diamonds (\$ per carat) are the blue, type IIb boron containing diamonds. While almost all diamonds are electrical insulators, the type IIb diamonds are electrically conductive and may be considered as possessing properties of semiconductor. Thanks to the boron impurities, the optical hue of these diamonds can range from light grey/blue to a dark steely-blue. Some famous examples of these blue natural diamonds are the Hope Diamond, the Blue Heart Diamond, the Blue Moon, the Wittelsbach-Graff Diamond, and others. Having the deep level boron impurity of relatively high concentration, these diamonds also show interesting fluorescence and phosphorescence properties when exposed to ultraviolet light and have been a subject of several recent investigations.[2-6]

Knowledge of the relative concentration of $\mathrm{N}$ and $\mathrm{B}$ in diamonds would be of great interest to understanding the competition in nature between those two elements during diamond growth. A detailed understanding of the electrical properties of boron in diamond is especially important for the exploitation of semiconducting diamonds for electronic applications.[7-11]

The purpose of this study was to comprehensively investigate the main properties of the electronic spectrum of rare, natural blue diamonds with moderate concentrations of boron. A non-destructive technique - a complex of temperature admittance spectroscopy (TAS) together with frequency admittance spectroscopy - was employed to study 
the electrical response of diamonds with boron. For semiconductor applications this measurement technique is more applicable than FTIR and dc electrical measurements traditionally used to inspect the quality of diamonds, as it tests physical properties of the crystal in conditions very close to operating regime of a microelectronic device, which possesses active, non-linear behaviors. The experimental apparatus used in this study was a purpose built computer-controlled admittance spectroscopy setup which covers a temperature range $20-450 \mathrm{~K}$ and frequency range $1 \mathrm{kHz}-2 \mathrm{MHz}$.[12-14]

By measurement and appropriate simulations, full characterization of electronic properties for boron impurity in 4 natural type IIb diamonds has been obtained, including the distribution of majority charge carrier concentrations and the thermionic emission characteristics.

\section{Methods of characterization}

The main measurement technique presented in this study is admittance spectroscopy. Admittance spectroscopy is the combination of conductance $(G)$ and capacitance $(C)$ measurements, carried out during one temperature cycle. By scanning different electric parameters of a $p$ - $n$ junction or Schottky barrier as function of temperature, applied bias $(V)$ and the frequency $\omega$ of the bias, one can propose a set of methods for quantitative characterization of semiconductor materials and structures, Fig. 1. Admittance spectroscopy allows one to characterize the following parameters for natural diamond samples: free charge carrier concentration, its distribution over the sample, the activation energy of deep level $E_{a}$, their capture cross section, emission and capture rates, their dependence on temperature and other parameters.

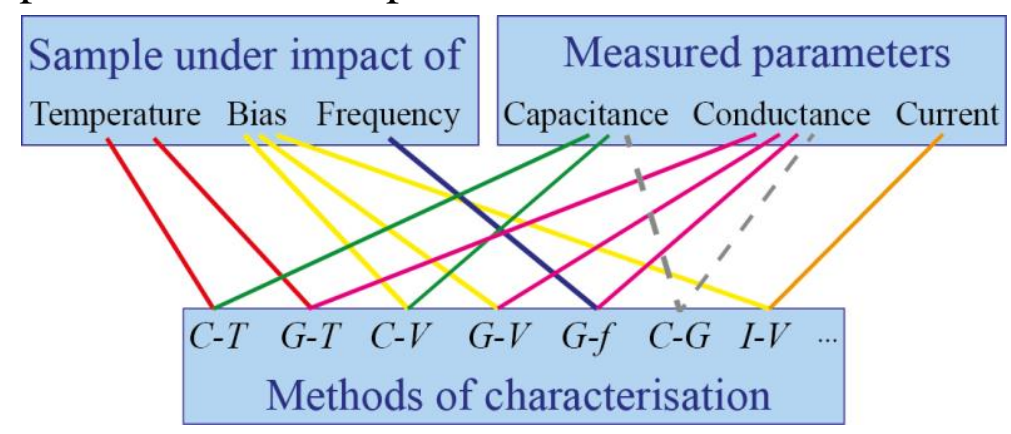

Fig. 1 Admittance techniques

Admittance measurements were carried out by means of a computer-controlled setup, the main parts of which are RLC-meter Agilent E4980A, closed-cycle helium probe station Janis CCR-10, and temperature controller LakeShore 336.[12-14]

A deep trap like boron in single crystal diamond can be characterized by an emission rate of charge carriers to the corresponding band (valence band in our case):[15]

$$
e_{p}=g_{A} v_{t h} \sigma_{p} N_{V} \exp \left(-E_{a} / k T\right)
$$


where $N_{\mathrm{V}}$ is the effective density of states, $v_{t h}$ is the thermal velocity for holes, $\sigma_{p}$ is the hole capture cross section, and $g_{A}$ is the degeneracy factor for the acceptor level. Taking in mind the temperature dependence of the pre-exponential factors, one usually rewrites (1) in the form

$$
e_{p}=A T^{2} \exp \left(-E_{a} / k T\right)
$$

where factor $A$ is independent on temperature.

When a small ac signal of frequency $\omega$ is applied to the sample with fabricated Schottky and Ohmic contacts, a trap with the emission rate (1) generates a conductance (and capacitance as well) response of the form:[16]

$$
G(T)=\frac{e_{p}(T) \omega^{2}}{e_{p}^{2}(T)+\omega^{2}} \frac{N_{T}}{p} S\left(\frac{\varepsilon \varepsilon_{0} e N_{A}^{-}}{2 V}\right)^{1 / 2} .
$$

In expression (3) $N_{\mathrm{T}}$ is the trap concentration, $p$ is the free charge carrier concentration, $\varepsilon$ is the relative dielectric constant for diamond, and $\varepsilon_{0}$ is the permittivity of vacuum. The square root term, multiplied by the Schottky contact area $S$, has the meaning of the sample barrier capacitance at the applied bias $V$. At the maximum of the conductance spectrum, the following relation is fulfilled:

$$
e_{p}=\omega \text {. }
$$

The processing of measured conductance spectra includes building the Arrhenius plots in coordinates $\ln \left(e / T^{2}\right)$ vs. $1 / T$ and derivation of apparent activation energy and capture cross section - the key characteristics of deep level. The term "apparent activation energy" here means the dynamic activation energy obtained in non-equilibrium capacitance or admittance measurements, in the presence of ac electric field. Together with the dynamic characteristics of the deep center, conductance spectra give (via $N_{\mathrm{T}} / p$ relation) the explicit information about the concentration of the trap under investigation.

Additional modeling of conductance (and capacitance) spectra according to (2) and (3) may reveal extra information about the possible energy broadening of the deep center.

Furthermore, for determination of free carrier concentration the capacitancevoltage $(C-V)$ profiling was used. The majority charge carrier (hole) concentration profile is obtained by derivation of measured $C-V$ characteristics:

$$
p_{C V}(w)=\frac{C^{3}}{e \varepsilon \varepsilon_{0} S^{2}}\left(\frac{d C}{d V}\right)^{-1} .
$$


At this, the charge depletion width, corresponding to the barrier capacitance at a given bias, is

$$
w=\frac{\varepsilon \varepsilon_{0} S}{C} .
$$

In case of the Schottky barrier the whole width is related to space charge region (SCR) of the semiconductor. Taking into account that the charge increment takes place only at the SCR edge, one can associate the width, derived from (6), with the current coordinate of the space charge region edge at a given bias, assuming the Schottky barrier location as a reference point $(x=0)$.

Note that $C-V$ profiling gives the concentration of free charge carriers in the sample. This is an even more important characteristic for a wide bandgap semiconductor like diamond $\left(\Delta E_{\mathrm{g}}=5.45 \mathrm{eV}\right)$ than the impurity concentration $N_{\mathrm{A}}$, because only free carriers (electrons and holes) produce the electric current through the semiconductor device, providing its desirable electronic properties. But, owing to the large activation energy of boron, only less than $1 \%$ of the impurity is ionized at room temperature).[17] Due to the peculiarities of the capacitance-voltage technique, the measured concentration of free charge carriers may differ from the impurity concentration, which will be further discussed in Section V.

\section{Samples under investigation}

A total of 4 natural blue type IIb diamonds were investigated in this study. The natural diamond samples were on loan from the Smithsonian Institution of Washington DC. The samples Apollo Blue and Steinmetz are double side polished. The sample Blue Pear (H26) is faceted as a gem. And the sample 111 has the major surfaces as cleaved $<111>$ surfaces.

FTIR measurements were performed both at the Smithsonian Institution and at University LETI. The estimated FTIR concentrations of boron are presented in Table 1.

Table 1. Characteristics of investigated samples

\begin{tabular}{|l|l|l|l|l|}
\hline & Steinmetz & Apollo Blue & 111 & Blue Pear \\
\hline $\begin{array}{l}\text { Uncomp. B from FTIR, ppm } \\
\text { recalculated in atoms } / \mathrm{cm}^{3}\end{array}$ & $\begin{array}{l}0.15-0.20 \\
(2-3) \cdot 10^{16}\end{array}$ & $\begin{array}{l}0.21-0.19 \\
3 \cdot 10^{16}\end{array}$ & $\begin{array}{l}0.09 \\
1.6 \cdot 10^{16}\end{array}$ & $\begin{array}{l}0.27-0.30 \\
(4.5-5) \cdot 10^{16}\end{array}$ \\
\hline$p$ from $C-V, \mathrm{~cm}^{-3}$ & $5 \cdot 10^{15}$ & $2.5 \cdot 10^{16}$ & $3.5 \cdot 10^{16}$ & $4 \cdot 10^{16}$ \\
\hline$G / \omega$ peaks ampl., pF & 1 & 2.3 & 3.5 & 2.1 \\
\hline Peak temperatures, K & $166-260$ & $176-270$ & $190-290$ & $180-280$ \\
\hline$E \mathrm{a}, \mathrm{meV} G-T(G-f)$ & $285 \pm 10$ & $322 \pm 2$ & $320 \pm 1$ & $315 \pm 1$ \\
\hline Factor $A, \mathrm{c}^{-1} \cdot \mathrm{K}^{-2}$ & $6.4 \cdot 10^{4}$ & $2.5 \cdot 10^{5}$ & $5.3 \cdot 10^{4}$ & $4.5 \cdot 10^{5}$ \\
\hline $\begin{array}{l}\text { Capture } \mathrm{cross} \\
\text { section }(\sigma), \mathrm{cm}^{2}\end{array}$ & $3.4 \cdot 10^{-14}$ & $1.5 \cdot 10^{-13}$ & $2.8 \cdot 10^{-14}$ & $2.4 \cdot 10^{-13}$ \\
\hline
\end{tabular}


The key challenge for admittance measurements is the fabrication of two types of contacts - Ohmic and Schottky. For wide bandgap diamond this is a very difficult task, and considerable time and effort were spent to work out the appropriate technology and geometry of contacts to achieve the best conditions for capacitance measurements.

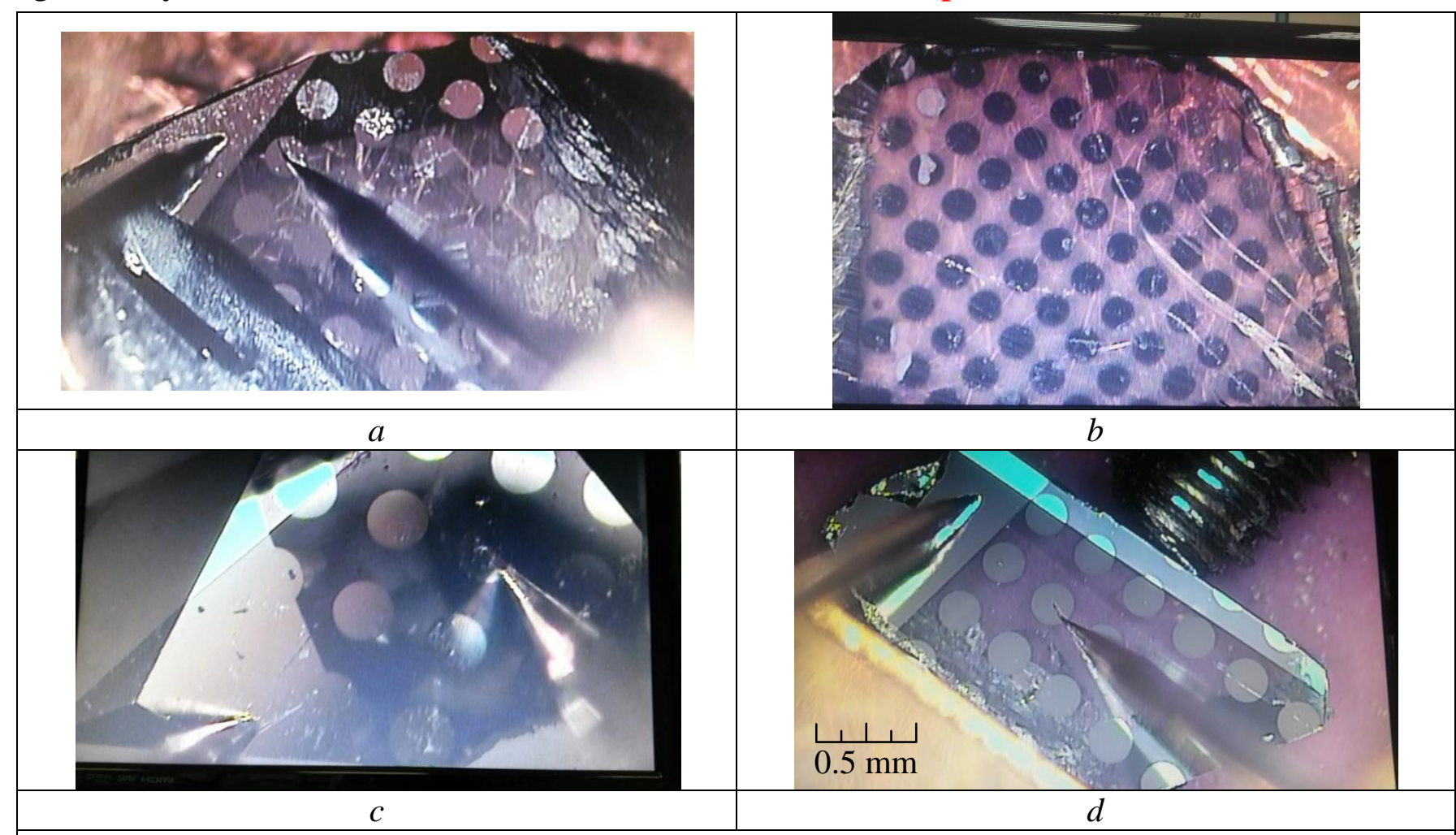

Fig.2 View of investigated samples with sputtered contacts and measuring probes: $a-$ Apollo Blue, $b$ - Diamond 111, $c$ - Blue Pear, $d$-Steinmetz. Scale bar is shown.

The side, to which both types of contacts were deposited, was chosen on the basis of surface quality inspection. The Ohmic and Schottky contacts for electrical investigation were deposited at different temperatures. The Pt large Ohmic contact (seen, for instance, as a wide strip near the edge of Steinmetz in Fig.2, $d$ ) was deposited at $T=300$ ${ }^{\circ} \mathrm{C}$, and smaller, round Pt Schottky contacts were deposited at $150{ }^{\circ} \mathrm{C}$. The diameters of the Schottky contacts were from 350 to $450 \mu \mathrm{m}$ from sample to sample, depending on the shape and cutting of the sample, Fig.2. The resulting contact area was taken into account in the concentration measurements. Special holders for samples Blue Pear and Steinmetz were fabricated, since their bottom sides are not flat.

\section{Experimental data}

\section{A. Capacitance-voltage profiling}

In order to examine the electrical properties of the samples, we used Schottky and Ohmic contacts. Measurements of their current-voltage $(I-V)$ characteristics were performed at various temperatures. At $T<180 \mathrm{~K}$ the boron impurity is practically unionized. The $I-V$ characteristic looks like that for a resistor. With temperature increasing the current-voltage characteristics become rectifying, and diamond possesses semicon- 
ductor properties. Typical $I-V$ characteristics for the Apollo Blue sample at various temperatures are presented in Fig.3. Rectification starts from about $+2 \mathrm{~V}$, and we saw very low leakage currents at reverse biases up to $10-20 \mathrm{~V}$. It is interesting that the minimum in $I-V$ characteristics was shifted from $U=0$ for $T<210 \mathrm{~K}$, at the temperatures where the emission from boron deep level begins to take place (see next Section). At present there is not a clear explanation for this, but we note that this is a deviation from wellknown Shockley theory.[18]

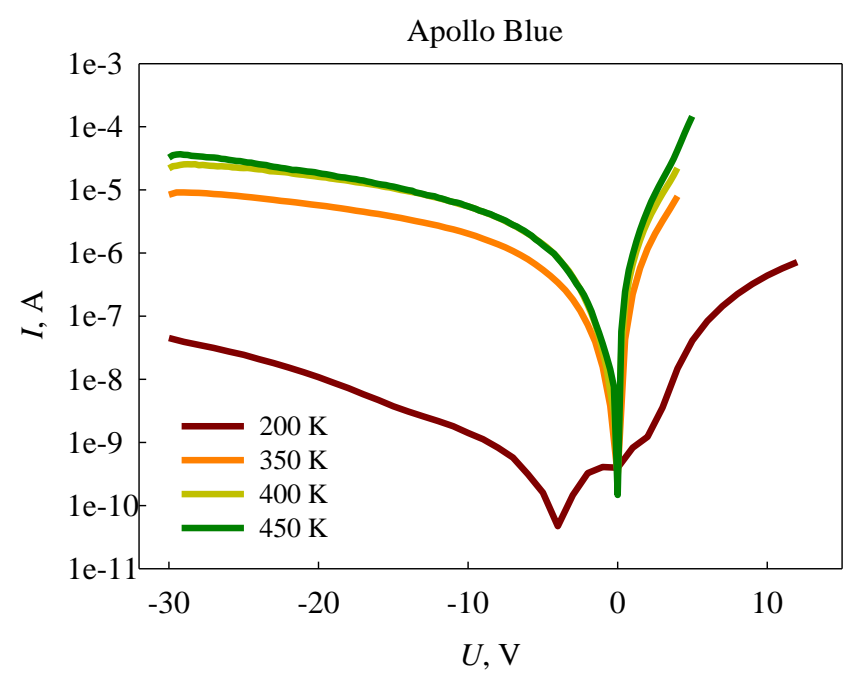

Fig.3 Typical $I$ - $V$ characteristics for the natural Apollo Blue diamond sample at various temperatures (abs value for $I$ is used)

Capacitance-voltage characteristics of the samples were measured at several test signal frequencies over a wide temperature range $(200-400 \mathrm{~K})$. The obtained $1 / \mathrm{C}^{2}-\mathrm{V}$ characteristics are linear in bias range from -10 to $0 \mathrm{~V}$. At forward biases there is significant current across the structure, and the RLC-meter cannot properly balance the measurement scheme.
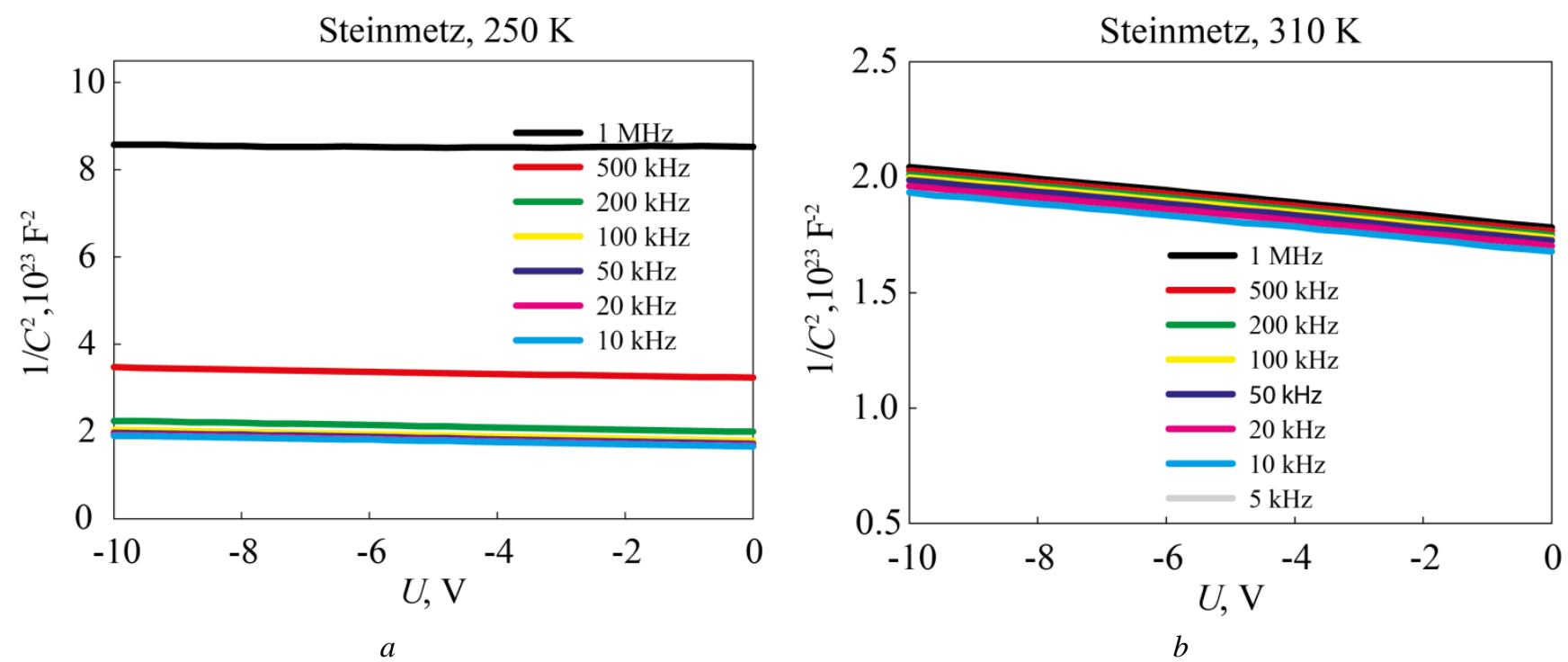

Fig. $41 / C^{2}-V$ characteristics for the Steinmetz blue diamond sample at: a) $250 \mathrm{~K}$ - non-quasistatic conditions;

b) $310 \mathrm{~K}$ - quasistatic conditions 
At relatively low temperatures $(<250 \mathrm{~K})$ a dependence of the capacitance-voltage characteristics on frequency is observed. This is the case of so-called non-quasistatic type of $C$ - $V$ measurements, Fig. $4 a$ vs. $4 b$.[15] This is due to the fact that the boron acceptor level is located deeply in the forbidden gap and is only partially ionized. The specific feature of the capacitance technique is the additional ionization of impurity with applied ac bias. The concurrence between the frequency of the induced ac bias and the deep center recharge velocity results in a frequency dependent ionization degree of the deep center under investigation. This leads to different values of charge and, hence, capacitance vs. test frequency, detectable in the experiment. Our experimental setup provides a wide range of test signal frequencies, thus controlling the ionization degree at a given temperature. At higher temperatures $(>300 \mathrm{~K}$ ), when quasistatic $C$ - $V$ measurements are fulfilled (Fig. $4 b$ ) for all examined samples, no frequency dispersion of capacitance is observed.

\section{B. Temperature and frequency admittance spectroscopy}

Measurements of temperature dependent conductance $(G / \omega-T)$ and capacitance $(C-T)$ spectra were carried out in the temperature range $20-450 \mathrm{~K}$ and frequency range $1 \mathrm{kHz}-2 \mathrm{MHz}$, Fig.5. Care was taken to obtain accurate measurements of diamond temperature, so the temperature ramp rate was as slow as $0.5 \mathrm{~K} / \mathrm{min}$. Temperature scanning was performed with rising and falling temperature, and the obtained spectra displayed practically no hysteresis.

Temperature conductance spectra at $U=0 \mathrm{~V}$ for all samples were, in general, quite similar. Their peak amplitudes $(G / \omega)$ did not depend on frequency and fell into the temperature interval $175-290 \mathrm{~K}$. We observed the permanent frequency transformation of a single peak, which belongs to a deep boron center.

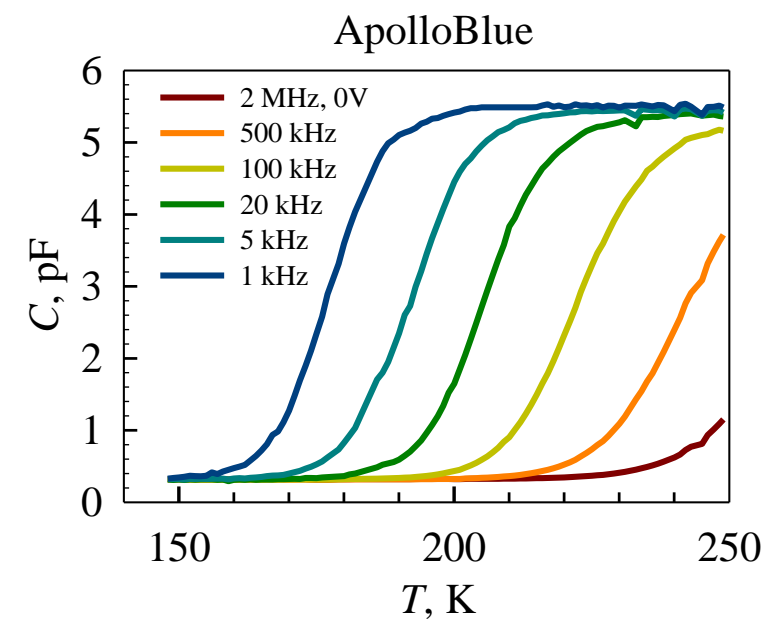

$a$

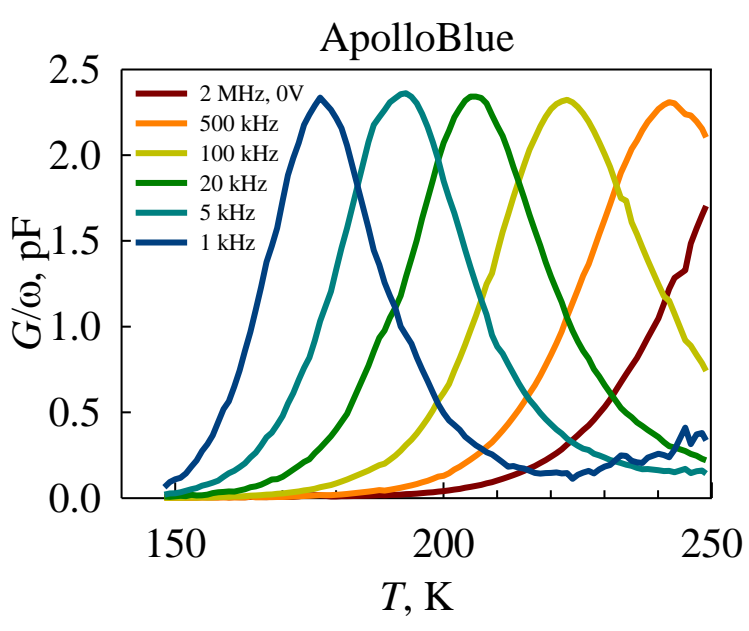

$b$

Fig.5 $C-T(a)$ and $G / \omega-T(b)$ spectra, Apollo Blue

An expected shift of conductance curves towards the higher temperatures with frequency increasing was observed - this is the distinctive feature of thermionic emis- 
sion. So, from the temperature admittance spectra, the activation energy of charge carriers from the impurity level can be evaluated in the usual manner via the Arrhenius plot, using the temperatures of conductance peak maxima,[15] (see Section V).

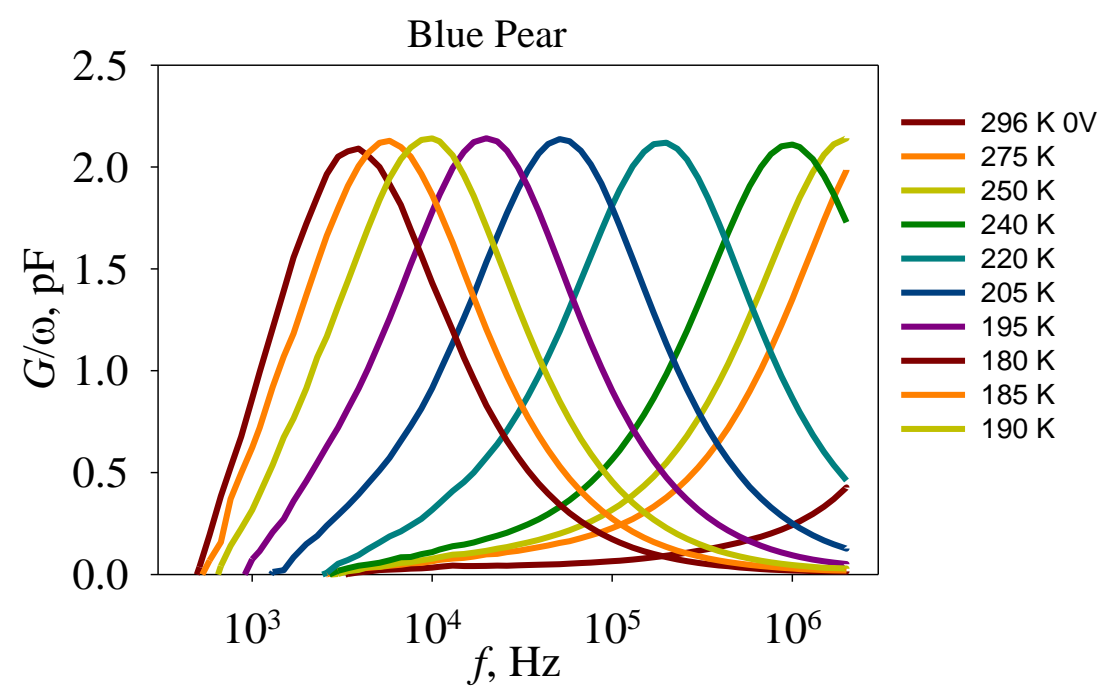

Fig.6 $G / \omega-f$ spectra for Blue Pear

Another measurement technique used in this study was frequency admittance spectroscopy. These measurements are performed by fixing the temperature and obtaining the capacitance and conductance response in intervals of frequency. The Arrhenius plots are created in similar manner using the frequencies of the $G / \omega-f$ peaks maxima. This technique is sometimes more convenient than temperature admittance spectroscopy, allowing one to obtain the same set of dynamic characteristics of the examined deep level. This technique was used to study the Apollo Blue and Blue Pear samples. The Blue Pear $G / \omega-f$ spectra were obtained in the whole RLC-device frequency range ( $1 \mathrm{kHz}$ $-2 \mathrm{MHz})$, Fig.6.

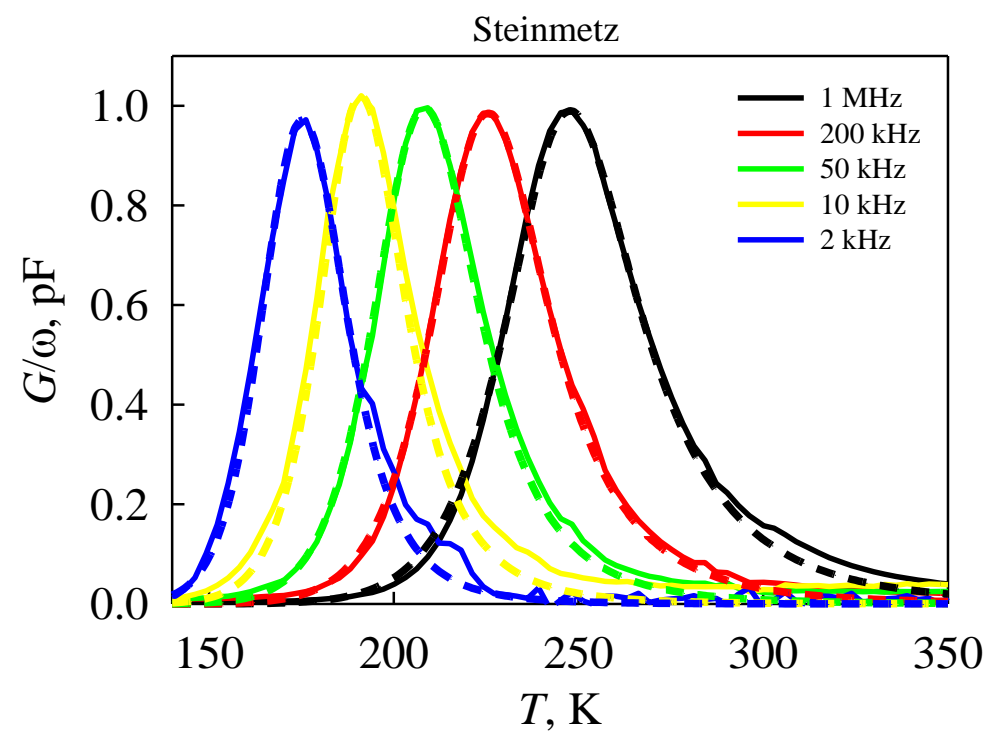

Fig.7 Experimental (solid line) and modeled (dashed line) $G / \omega$ vs. $T$ spectra for Steinmetz sample at various frequencies 
The Steinmetz diamond exhibited slightly different behavior. Its peaks in conductance spectra are shifted towards low temperatures and the peak maxima drop to about 1 pF, Fig.7. Such a decrease in conductance is in good agreement with the obtained hole concentration for this sample, which is smaller than for others.

\section{DISCUSSION}

\section{A. Carrier concentration profiles}

The carrier (hole) concentration profiles at various temperatures for the examined samples were obtained via derivation of $\mathrm{C}-\mathrm{V}$ characteristics. Note, that this method measures the thermionic activation of the dopant at the edge of the space charge region (SCR).

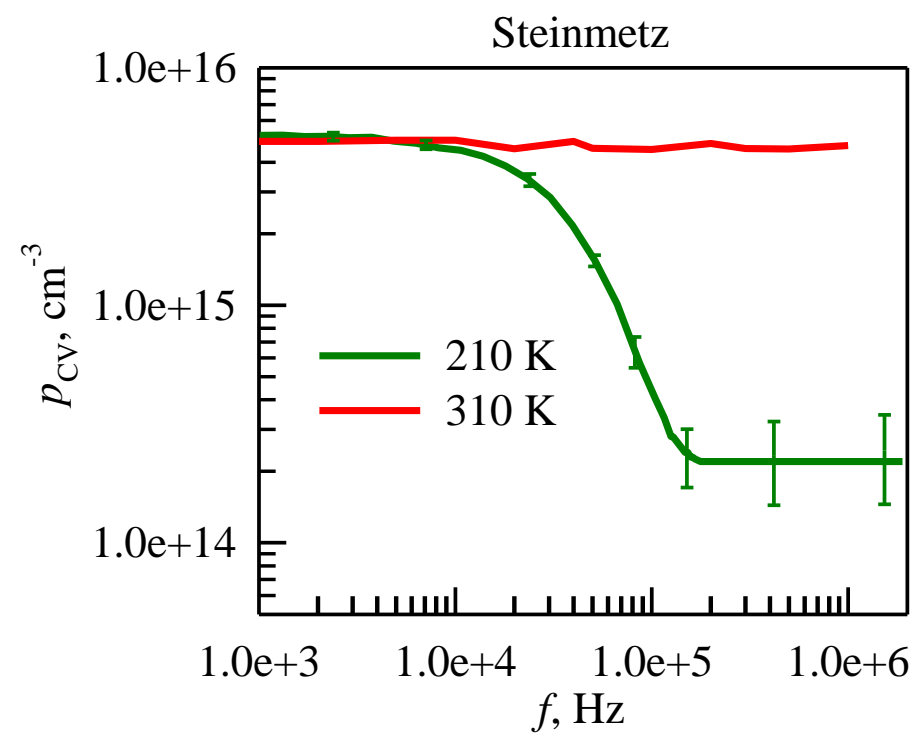

Fig. 8 Apparent concentration vs. frequency for Steinmetz sample; $T=210$ and $310 \mathrm{~K}$. Error bars are shown

The dependence of net hole concentration on signal frequency was investigated for samples Steinmetz and Apollo Blue at various temperatures. At relatively low temperatures $(<250 \mathrm{~K})$, when the $C-V$ characteristics are non-quasistatic and the boron acceptor level is only partially ionized, even in the presence of additional ac field, we see strong dependence of apparent carrier concentration on frequency, see Fig. 8 for the Steinmetz. At higher temperatures $(>300 \mathrm{~K})$ the quasistatic $C$ - $V$ measurement conditions are fulfilled (see Fig. $4 b$ ), and the derived carrier concentration $p_{\mathrm{CV}}$ is independent of frequency. Similarly for the Apollo Blue sample, the average concentration of charge carriers at high temperatures is higher (about $2.5 \cdot 10^{16} \mathrm{~cm}^{-3}$ ). At higher frequencies the apparent $C$ - $V$ concentration falls down to $1 \cdot 10^{14} \mathrm{~cm}^{-3}$ for both samples. 




Fig.9 Carrier concentration profiles at different temperatures, Blue Pear diamond

At temperatures $>T_{\text {Room }}$ the calculated concentration profiles for Apollo Blue, Blue Pear and 111 diamonds are almost the same - we see small variation of concentration from $2 \cdot 10^{16} \mathrm{~cm}^{-3}$ to $4 \cdot 10^{16} \mathrm{~cm}^{-3}$ while the space charge region width is about $1.35-$ $1.50 \mu \mathrm{m}$, (Fig.9 for Blue Pear). In general, these data are in good agreement with the uncompensated boron concentration obtained by FTIR.

For Steinmetz, the $p_{\mathrm{CV}}$ concentration is $(4.5-5) \cdot 10^{15} \mathrm{~cm}^{-3}$ at the maximum ionization and decreases to $2 \cdot 10^{14} \mathrm{~cm}^{-3}$ at high frequencies and reduced temperatures. The concentration slightly increases with depth from $3 \cdot 10^{15}$ to $5 \cdot 10^{15} \mathrm{~cm}^{-3}$, the corresponding coordinate (space charge region width) sweeps from 2.7 to $3.0 \mu \mathrm{m}$. We found that the charge carrier concentration for Steinmetz is less than for other investigated samples, and the obtained charge carrier concentration doesn't match with the FTIR measurement for uncompensated boron.

The average hole concentrations obtained from the $\mathrm{C}-\mathrm{V}$ characteristics for all the samples are presented in Table 1. They are in reasonable coincidence with FTIR results.

\section{B. Activation energies}

The Arrhenius plots for all samples were created using the temperatures (for Steinmetz, Apollo Blue, 111) or frequencies (for Apollo Blue and Blue Pear) of conductance peak maxima. The apparent activation energy of charge carriers from the impurity level was obtained according to (2) as a slope of the line plotted in coordinates $\ln \left(e / T^{2}\right)$ vs. 1000/T, and the capture cross section via the factor $A$ in (1) - (2). The activation energies and capture cross sections are presented in Table 1. For samples Apollo Blue and 111 the experimental points of the Arrhenius plots are fit linearly with high accuracy for the entire temperature range,

Fig. 10. For these samples the Arrhenius plots give practically the same activation energies $322 \pm 2$ and $320 \pm 1 \mathrm{meV}$; the capture cross section are $1.5 \cdot 10^{-13} \mathrm{~cm}^{2}$ and $2.2 \cdot 10^{-13}$ 
$\mathrm{cm}^{2}$, respectively. The derived activation energies were the same for conductance measurements with increasing and decreasing temperatures.

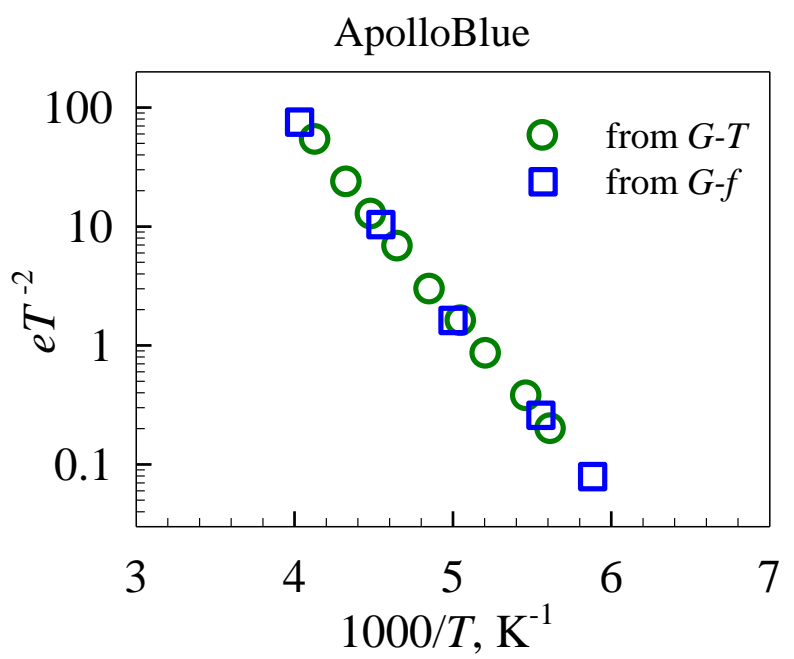

Fig. 10 Arrhenius plots for Apollo Blue from G-T and G-f spectra

It is important that the points of the Arrhenius plot from $G / \omega-f$ measurements are in good coincidence with those for $G / \omega-T$ measurements,

Fig. 10, and the apparent activation energies, obtained from $G-T$ and $G-f$ measurements for Apollo Blue, are not significantly different.

The Arrhenius plots for all 4 samples are shown in Fig.11. It is seen that the Arrhenius plots for Apollo Blue, Diamond 111 and Blue Pear are nearly parallel to each other, as their apparent activation energies are almost the same - between $315 \mathrm{meV}$ (for Blue Pear) and $325 \mathrm{meV}$ (Apollo Blue, Diamond 111). The shifts among their Arrhenius plots apparently originate from differences in capture cross sections.

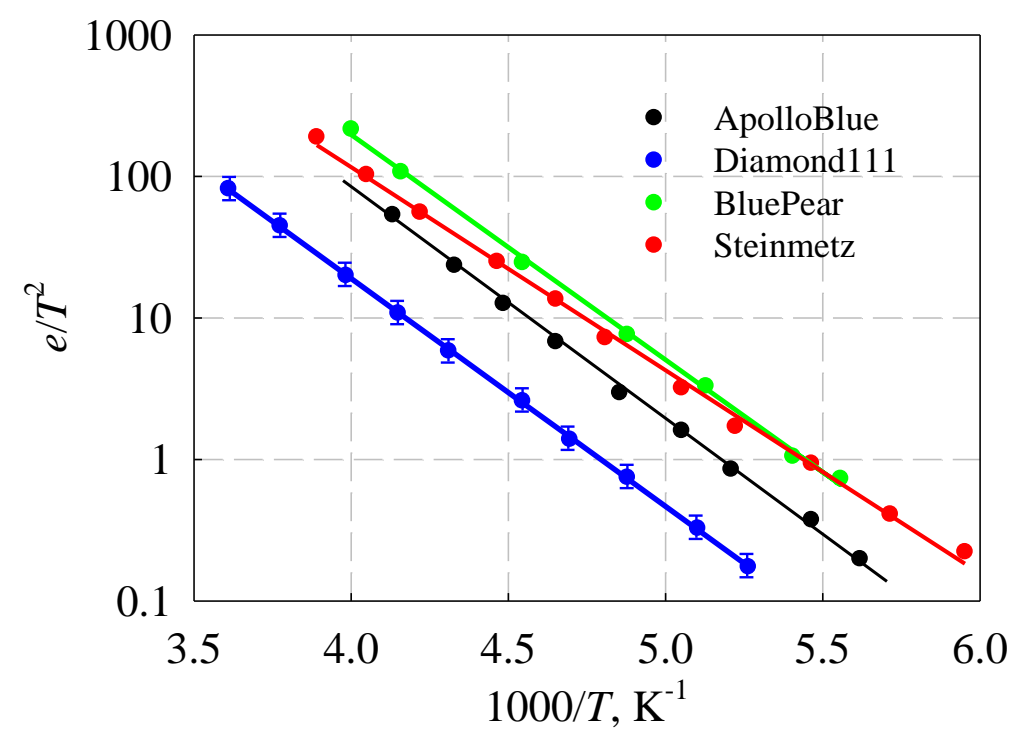

Fig.11 Arrhenius plots for all samples. Error bars are shown.

The slope of Arrhenius plot for Steinmetz is significantly different, resulting in $E_{\mathrm{A}}$ $\sim 285 \pm 10 \mathrm{meV}$. Its capture cross section is several times less. 
Note, that the activation energies obtained for the natural diamond samples are similar to those measured for boron doped CVD grown diamond samples with $\mathrm{B} / \mathrm{C}<600 \mathrm{ppm}$.[17] As it can be seen, the mean square error (MSE) for the activation energy of the natural boron doped diamonds is less than $2 \mathrm{meV}$. One should note that the $E_{\mathrm{A}} \mathrm{MSE}$ is the criterion of crystal perfection, and for the CVD-epitaxial diamond layers, which we studied previously, the MSE was 2-3 times larger.

We have simulated the temperature conductance spectra for samples using the measured activation energies and factor $A$ values from Table 1 . The calculated curves fit very well the experimental spectra, and the possible broadening is less than 1\%, Fig.7.

The optical properties of the boron acceptor level are well known and give an optical ionization energy for the boron acceptor of $373 \mathrm{meV}$. [19] In this study, we have measured with high precision the apparent activation energy of boron acceptor as between 315 and $325 \mathrm{meV}$ for three natural diamond samples - Apollo Blue, Blue Pear, and Diamond 111. This is significantly lower than the optical ionization energy $E_{A}$, and also lower than the thermal activation energy of $0.37 \mathrm{eV}$ determined by dc electrical transport measurements on natural and synthetic type IIb diamonds (see, for instance $[20,21])$. The reason for the difference is the physics underlying the different measurement techniques.

The free charge carrier concentration, measured using the dc electrical transport technique with ohmic contacts, corresponds to the well-known expression for partially ionized acceptor impurity $N_{A}^{-}$:

$$
N_{A}^{-}=\frac{N_{A}}{1+\left(1 / g_{A}\right) \exp \left[\left(E_{A}-E_{F}\right) / k T\right]},
$$

where $g_{A}$ is the degeneracy factor for boron, and $E_{F}$ is the Fermi level. The admittance method, in contrast to dc electrical measurements of conductivity, is carried out under non-equilibrium conditions (due to the applied electric field) and is based on the resonant nature of the measurements. Here the ionized concentration is registered at ac frequency in vicinity of the space change region edge (that is coordinate dependent) and has an additional ionization, forced by the ac test signal:

$$
N_{A}^{-}(x)=\frac{N_{A}}{1+\left(1 / g_{A}\right) \exp \left[\left(E_{A}-E_{F}-e \varphi_{d c}(x)-e \varphi_{a c}(x)\right) / k T\right]} .
$$

Here $\varphi_{d c}(x)$ is the dc potential applied to the sample by the dc bias (it is used for shifting the point of observation); the test signal of RLC-device produces, in turn, the ac part of potential $\varphi_{a c}(x)$. Here $x$ is the distance from the Schottky barrier.

One may rewrite the expression in the form 


$$
N_{A}^{-}(x)=\frac{N_{A}}{1+\left(1 / g_{A}\right) \exp \left[\left(E_{A}-E_{F}\right) / k T\right] M(x)} .
$$

Here the factor $M(x)=\exp \left[\left(-e \varphi_{d c}(x)-e \varphi_{a c}(x)\right) / k T\right]$ is coordinate-dependent and divides the sample into three parts: 1) the space charge region near the rectifying barrier, where the impurity is totally ionized (small $x$ ); 2) an intermediate region where the acceptor level crosses the Fermi level; and 3) the electroneutrality region, where $M(x)$ approaches to 1 and the equilibrium carrier distribution described by (7) takes place. The ac part of potential $\varphi_{a c}(x)$, acting in the intermediate region, causes here the recharging of the deep boron center and produces the ac current in the outer circuit, proportional to the local carrier concentration at the edge of SCR.

The degree of compensation for the Apollo Blue and the Blue Pear (H26) can be estimated from the previously reported values measured by time-of-flight secondary ion mass spectroscopy (ToF SIMS) and FTIR absorption, see Table 5 in [2]. In that study, the ToF SIMS measurements were conducted on micron sized areas and gave a large variability from point to point, while the FTIR measurement was averaged over mm sized areas. It is not surprising to find significant inhomogeneities in natural diamond due to their often complicated growth history. Yet comparison of the uncompensated boron, measured by FTIR, and the total boron, measured by SIMS, suggests that the Apollo Blue and the Blue Pear (H26) are much less compensated than the Steinmetz sample.

\section{CONCLUSIONS}

For the first time precision admittance measurements were carried out for a set of four rare, natural type IIb diamond samples in wide temperature and frequency ranges. The peaks in temperature and frequency conductance spectra were used to compute the Arrhenius plots, and the apparent activation energies for boron deep level were obtained from these plots. It was shown that $G / \omega-T$ and $G / \omega-f$ spectra give the same activation energies. Three samples (ApolloBlue, Diamond 111 and Blue Pear) showed quite similar conductance spectra, and the obtained activation energies for these samples were $320 \pm 2$ $\mathrm{meV}$ and $315 \pm 5 \mathrm{meV}$. These energies correspond to low doped CVD-grown diamond samples with $\mathrm{B} / \mathrm{C}<600 \mathrm{ppm}$. Capture cross sections were generally about $10^{-13} \mathrm{~cm}^{2}$. For the Steinmetz sample the apparent activation energy is $285 \pm 10 \mathrm{meV}$, and its capture cross section is several times less.

The capacitance-voltage profiling was used to estimate the majority charge carrier concentration. Obtained carrier concentrations are in the range $(1-5) \cdot 10^{16} \mathrm{~cm}^{-3}(0.06-0.3$ ppm) for three of the samples, and are in good coincidence with FTIR results. For the Steinmetz sample, the concentration is considerably less - about $4 \cdot 10^{15} \mathrm{~cm}^{-3}$. 


\section{ACKNOWLEDGEMENTS}

This work was supported by Act 220 of the Russian Government (Agreement No. 14.B25.31.0021 with the host organization IAP RAS). The Steinmetz blue sample was generously provided to the Smithsonian Institution by the Steinmetz Company.

The authors give thanks to Dr. D. Chigirev for contacts fabrication, Dr. O. Kucherova for assistance in admittance measurements, and Dr. M. Panov (all from LETI) for FTIR measurements. They also thank Prof. Alan Collins (King's College London, UK) for valuable and useful discussions.

\section{REFERENCES}

[1] J.E. Field, The Properties of Natural and Synthetic Diamond, Academic Press, 1992. [2] E. Gaillou, J.E. Post, D. Rost, J.E. Butler, Boron in natural type IIb blue diamonds: Chemical and spectroscopic measurements, American Mineralogist, 97 (2012) 1-18. [3] S. Eaton-Magana, J.E. Post, P.J. Heaney, J. Freitas, P. Klein, R. Walters, J.E. Butler, Using phosphorescence as a fingerprint for the Hope and other blue diamonds, Geology, 36 (2008) 83-86.

[4] E. Gaillou, J.E. Post, K.S. Byrne, J.E. Butler, STUDY OF THE BLUE MOON DIAMOND, Gems \& Gemology, 50 (2014) 280-286.

[5] E. Gaillou, D. Rost, J.E. Post, J.E. Butler, Quantifying boron in natural type IIb blue diamonds, Geochimica et Cosmochimica Acta, 74 (2010) A313-A313.

[6] E. Gaillou, W.Y. Wang, J.E. Post, J.M. King, J.E. Butler, A.T. Collins, T.M. Moses, THE WITTELSBACH-GRAFF AND HOPE DIAMONDS: NOT CUT FROM THE SAME ROUGH, Gems \& Gemology, 46 (2010) 80-88.

[7] R.S. Balmer, I. Friel, S.M. Woollard, C.J.H. Wort, G.A. Scarsbrook, S.E. Coe, H. ElHajj, A. Kaiser, A. Denisenko, E. Kohn, J. Isberg, Unlocking diamond's potential as an electronic material, Philosophical Transactions of the Royal Society a-Mathematical Physical and Engineering Sciences, 366 (2008) 251-265.

[8] Y. Gurbuz, O. Esame, I. Tekin, W.P. Kang, J.L. Davidson, Diamond semiconductor technology for RF device applications, Solid-State Electronics, 49 (2005) 1055-1070. [9] K. Thonke, The boron acceptor in diamond, Semiconductor Science and Technology, 18 (2003) S20-S26.

[10] J.E. Butler, M.W. Geis, K.E. Krohn, J. Lawless, S. Deneault, T.M. Lyszczarz, D. Flechtner, R. Wright, Exceptionally high voltage Schottky diamond diodes and low boron doping, Semiconductor Science and Technology, 18 (2003) S67-S71.

[11] N. Tumilty, J. Welch, R. Lang, C. Wort, R. Balmer, R.B. Jackman, An impedance spectroscopic investigation of the electrical properties of delta-doped diamond structures, Journal of Applied Physics, 106 (2009).

[12] V.I. Zubkov, I.N. Yakovlev, V.G. Litvinov, A.V. Ermachihin, O.V. Kucherova, V.N. Cherkasova, Analysis of the electrostatic interaction of charges in multiple InGaAs/GaAs quantum wells by admittance-spectroscopy methods, Semiconductors, 48 (2014) 917-923. 
[13] O.V. Kucherova, V.I. Zubkov, E.O. Tsvelev, I.N. Yakovlev, A.V. Solomonov, Nondestructive Diagnostics of Nanoheterostructures with InGaN/GaN Multiple Quantum Wells by Thermal Admittance Spectroscopy, Inorganic Materials, 47 (2011) 1574-1578.

[14] A.I. Zubkov, I.N. Yakovlev, O.V. Koucherova, Y.A. Orlova, Admittance Spectroscopy as a Method for Investigating Relaxation Processes in Quantum-Sized Heterostructures, Bulletin of the Russian Academy of Sciences, Physics, 75 (2011) 1406-1412.

[15] P. Blood, J.W. Orton, The electrical characterization of semiconductors: majority carriers and electron states, Academic Press, London, 1992.

[16] G. Vincent, D. Bois, P. Pinard, CONDUCTANCE AND CAPACITANCE STUDIES IN GAP SCHOTTKY BARRIERS, Journal of Applied Physics, 46 (1975) 5173-5178.

[17] V.I. Zubkov, O.V. Kucherova, S.A. Bogdanov, A.V. Zubkova, J.E. Butler, V.A. Ilyin, A.V. Afanas'ev, A.L. Vikharev, Temperature admittance spectroscopy of boron doped chemical vapor deposition diamond, Journal of Applied Physics, 118 (2015) 9. [18] S.M. Sze, K.K. Ng, Physics of Semiconductor Devices, 3rd Edition, Wiley Interscience, Hoboken, NJ, 2006.

[19] J. Walker, Optical absorption and luminescence in diamond, Reports on Progress in Physics, 42 (1979) 1605-1659.

[20] A.T. Collins, Lightowl.Ec, PHOTOTHERMAL IONIZATION AND PHOTONINDUCED TUNNELING IN ACCEPTOR PHOTOCONDUCTIVITY SPECTRUM OF SEMICONDUCTING DIAMOND, Physical Review, 171 (1968) 843-\&.

[21] Lightowl.Ec, A.T. Collins, ELECTRICAL-TRANSPORT MEASUREMENTS ON SYNTHETIC SEMICONDUCTING DIAMOND, Physical Review, 151 (1966) 685-\&. 